\title{
LA CLASSE GRAMMATICALE A-T-ELLE UNE INFLUENCE DANS L'APPARITION DES DISFLUENCES PERÇUES COMME SEVERES ? UNE ETUDE SUR LE FRANÇAIS ET LE SLOVAQUE
}

Ivana DIDIRKOVA

F.R.S. - FNRS \& Université catholique de Louvain

Agnès STEUCKARDT

UMR 5267 Praxiling \& CNRS, Université Paul-Valéry Montpellier 3

Fabrice HIRSCH

UMR 5267 Praxiling \& CNRS, Université Paul-Valéry Montpellier 3

\begin{abstract}
En): This paper deals with morpho-syntactic characteristics of stuttering-like disfluencies. More specifically, the aim of this research was to observe the linguistic features of sequences where a prolongation, a block or a repetition is present and to verify if the results are similar in two languages. To carry out this research, 20 persons who stutter (10 Slovak-speaking and 10 French-speaking) were recorded. Recordings were transcribed in Phon and TreeTagger was used to obtain grammatical classification of the disfluent elements. Our results show several differences between the two groups.
\end{abstract}

Keywords (En): stuttering; disfluencies; grammatical characteristics; French; Slovak

Mots-clés (Fr) : bégaiement ; disfluences ; caractéristiques grammaticales ; Français ; Slovaque

\section{Introduction}

\subsection{Bégaiement et linguistique}

« Dans une grande partie des productions orales, les locuteurs improvisent leurs discours, et les marques de cette improvisation (...) sont nombreuses : répétitions, ratures, recherches de mots, hésitations, « euh », inachèvements, etc. » (BLANCHEBENVENISTE, 1991).

Comme le suggère la citation de BLANCHE-BENVENISTE (1991), la production d'énoncés oraux se fait rarement sans éléments à l'origine d'une rupture de la fluence. Ces accidents de parole, nommés également disfluences, sont normaux car ils sont le signe que les capacités du locuteur à émettre un message sont momentanément inférieures aux exigences linguistiques nécessaires pour le produire (STARKWEATHER, 1987). Dans la parole normo-fluente, ces disfluences passent généralement inaperçues. Toutefois, il arrive que ces accidents soient davantage apparents, comme dans le cas de certains troubles de la parole tels que le bégaiement.

Le bégaiement est une altération de la fluence de la parole qui a des conséquences négatives sur la communication (MONFRAIS-PFAUWADEL, 2014). Il se caractérise par la présence d'accidents de parole sévères qui empêchent la personne qui en souffre de s'exprimer de manière fluide. Si la plupart des études actuelles concluent que l'emplacement des disfluences dépend avant tout de facteurs 
moteurs (MONFRAIS-PFAUWADEL, 2014 ; DIDIRKOVA, 2016), un certain nombre de chercheurs ont tenté d'établir un lien entre le risque de voir apparaître une disfluence et d'autres niveaux linguistiques.

Ces recherches ont notamment montré que les mots lexicaux étaient davantage bégayés que les mots grammaticaux chez l'adulte qui bégaie (ROMMEL, 2001 ; AUYEUNG et al., 2003; DWORZYNSKI et al., 2003). Précisons que les mots grammaticaux sont les unités qui contribuent à l'organisation grammaticale de la phrase. Ils comprennent les prépositions, les pronoms, les conjonctions, les déterminants et les verbes auxiliaires. Les mots lexicaux regroupent les substantifs, les verbes, les adjectifs et les adverbes.

Pour expliquer les résultats évoqués plus haut, DWORZYNSKI et al. (2003) se sont penchés sur le niveau de difficulté des mots ayant subi une disfluence. Leur étude montre qu'une augmentation du « factor-value » (i.e. du niveau de difficulté) des mots a pour conséquence une hausse du nombre de bégayages.

L'emplacement du mot dans la phrase pourrait également avoir une influence sur la perte de fluence chez les personnes qui bégaient (désormais PQB). En effet, selon BLOODSTEIN et GANTWERK (1967), ou plus récemment BUHR et ZEBROWSKI (2009), les mots lexicaux seraient plus touchés par des bégayages lorsqu'ils se trouvent en position non initiale, alors que l'inverse est observé pour les mots grammaticaux, ces derniers étant davantage disfluents en position initiale dans la phrase.

OZDEMIR et al. (2015) ont entrepris de mener une étude sur le type de mots bégayés en turc. Partant du fait qu'il s'agit d'une langue agglutinante qui appartient à la famille ouralo-altaïque et qu'elle est donc différente de par sa structure de langues comme l'anglais, il se pourrait que les résultats ne soient pas identiques à ceux obtenus pour les langues indo-européennes. Les trois chercheurs soulignent notamment un ordre de mots relativement libre dans la syntaxe de la phrase, ainsi qu'un système flexionnel relativement riche. Leurs observations montrent que malgré des différences dans les structures linguistiques, les structures bégayées sont identiques à celles relevées pour les langues indo-européennes en ce que les adultes bégaient davantage sur les mots lexicaux (16\%) que sur les mots-outils (5\%). Ces résultats sont cependant en contradiction avec ceux obtenus par ABDALLA et al. (2010), qui ne relèvent aucune différence entre les mots-outils et les mots lexicaux en termes de fréquence de bégayages en arabe. OZDEMIR et al. (2015) notent pourtant que les mots pouvant être considérés à la fois comme grammaticaux et comme lexicaux semblent les plus problématiques.

D'autres études se sont fondées sur le lien entre la nature grammaticale d'un mot et sa réalisation orale, en tenant compte de l'accentuation (BLOODSTEIN \& GANTWERK, 1967 ; WINGATE, 1979 ; HOWELL et al., 1999 ; NATKE et al., 2004). Ces travaux concluent qu'une relation peut effectivement être établie entre l'accent primaire et la distribution des bégayages en prenant en considération la catégorie lexicale du mot.

PRINS et al. (1997) se sont, quant à eux, intéressés aux catégories grammaticales les plus souvent bégayées. Leur étude a dévoilé que les PQB produisent davantage de disfluences sur les verbes que sur les substantifs. Ce résultat fait écho aux travaux de QUARRINGTON et al. (1962, in NEWMAN \& BERSTEIN-RATNER, 2007) qui ont 
constaté que les substantifs constituent la classe grammaticale la moins disfluente parmi tous les mots lexicaux en parole conversationnelle.

Par conséquent, ces différents travaux montrent que la littérature portant sur le bégaiement s'est également intéressée aux aspects linguistiques de ce trouble. Cela étant, un certain nombre d'observations contradictoires ont été relevées. En outre, la disparité des résultats observés peut également s'expliquer par les langues étudiées, raison pour laquelle il semble important de prolonger les recherches menées jusqu'à présent sur d'autres systèmes linguistiques.

\subsection{Objectif et hypothèses}

$\mathrm{Au}$ vu des résultats souvent divergents obtenus dans la littérature, notre objectif est de vérifier s'il existe des régularités dans les structures linguistiques touchées par les bégayages. Nous nous concentrerons dans cette étude sur les types de mots (mots-outils et mots lexicaux) dans deux langues différentes, le français et le slovaque. Ensuite, nous nous intéresserons de plus près aux catégories grammaticales concrètes.

Notre hypothèse est que les deux langues étudiées devraient se comporter comme d'autres langues sur lesquelles ce type d'études a été mené, à savoir que les mots lexicaux devraient être bégayés davantage que leurs homologues grammaticaux. Nous pensons en effet que la longueur, la structure phonétique et la fréquence moins élevée de ces derniers devraient favoriser l'apparition d'un nombre plus important de disfluences. Pour ce qui est des catégories grammaticales, nous supposons que parmi les mots bégayés, nous devrions retrouver notamment des verbes et des substantifs en tant que porteurs de l'information principale. De même, nous supposons que les déterminants (en français) ainsi que les pronoms devraient être bégayés, étant donné qu'ils sont souvent présents en début d'énoncé, ce qui est peut-être problématique pour les PQB.

\section{Méthodologie}

La parole de 10 personnes qui bégaient slovacophones et de dix locuteurs francophones atteints de ce même trouble a été analysée dans le cadre de cette étude. Les deux groupes de locuteurs étaient appariés en sexe, en âge et en fonction de la sévérité de leur bégaiement. Au total, 6 femmes et 14 hommes ont participé à l'étude, tous adultes et monolingues.

Les enregistrements, réalisés à l'aide d'un enregistreur numérique donnant des fichiers au format.wav, se sont déroulés dans un endroit calme et isolé. Deux tâches ont été demandées aux locuteurs : dans un premier temps, ces derniers devaient lire la fable Le lion et le rat dans leur langue respective. Il a été demandé aux sujets de s'installer confortablement et de démarrer la lecture à haute voix sans prendre connaissance du texte auparavant. Ainsi, aucun des locuteurs, qu'ils soient francophones ou slovacophones, n'était familiarisé avec l'extrait à lire et sa structure grammaticale avant de commencer à produire la tâche demandée. Dans un second temps, les sujets avaient pour consigne de décrire une journée-type afin d'obtenir de la parole spontanée. En cas de besoin, ils étaient relancés par l'expérimentateur, l'objectif étant d'obtenir en moyenne sept minutes de production orale en dehors de la parole lue. Notons que le choix de travailler avec de la parole 
lue a été motivé par la volonté de contrôler la distribution des différentes unités étudiées dans les productions orales des locuteurs.

Les fichiers .wav obtenus à partir desdits enregistrements ont été exportés dans le logiciel Phon (Rose et al., 2016) et transcrits orthographiquement. Par la suite, une annotation des disfluences a été effectuée manuellement. Signalons que seules les disfluences perçues comme typiques du bégaiement ont été annotées; les disfluences dites « normales » n'ont pas été prises en compte. Nous avons travaillé avec trois types de bégayages : les répétitions, les prolongations et les blocages. Afin qu'un accident de parole puisse être perçu comme typique du bégaiement, nous nous sommes fondés sur les critères acoustico-perceptifs tels que décrits dans DIDIRKOVA et al. (2016).

$\mathrm{Au}$ total, 250 disfluences typiques du bégaiement ont été relevées en parole lue, dont 78 en français et 172 en slovaque. En parole spontanée, l'étude porte sur 628 bégayages produits par les locuteurs francophones et 602 par les sujets slovacophones, ce qui donne un total de 1230 disfluences.

L'ensemble des transcriptions a ensuite été exporté dans TreeTagger (SCHMID, 1995) dans l'objectif d'identifier les catégories grammaticales des mots produits par les sujets. Après vérification manuelle, les mots ont également été répartis en deux catégories : les mots-outils et les mots lexicaux. Les mêmes annotations ont été faites sur les mots bégayés uniquement et ce, afin de déterminer les catégories les plus touchées par les bégayages. Les résultats présentés dans la partie suivante sont systématiquement donnés en pourcentages de mots bégayés sur les mots produits. En d'autres termes, la proportion de substantifs bégayés, par exemple, est calculée par rapport au nombre total de substantifs produits.

Enfin, la robustesse des résultats a été vérifiée à l'aide du test exact de Fisher.

\section{Résultats}

Signalons que les résultats seront présentés pour les deux langues séparément. De même, nous décrirons dans un premier temps les résultats obtenus en lecture avant de continuer avec ceux obtenus en parole spontanée.

\subsection{Français}

Il est à signaler que la sévérité moyenne (exprimée en pourcentage d'occurrences bégayées par rapport aux occurrences prononcées) est moindre chez les sujets francophones en situation de lecture qu'en tâche de parole spontanée : elle atteint $6,66 \%(e ́-t 5,34 ;$ val. min 0 , val. $\max 13,68)$. Deux sujets sur les dix ayant participé à l'étude n'ont produit aucun bégayage lorsqu'ils lisaient la fable proposée.

\subsubsection{Lecture}

Le texte lu par les locuteurs francophones comprend 59 mots grammaticaux et 58 mots lexicaux, faisant des totaux respectifs de 590 mots grammaticaux et de 580 mots lexicaux prononcés par l'ensemble des locuteurs, soit 1170 mots. Sur ce total, 78 ont subi une disfluence sévère : 44 disfluences ont porté sur des mots lexicaux (représentant ainsi 7,76\% d'occurrences) et 34 sur des mots grammaticaux $(5,60 \%$ de toutes les occurrences). Ainsi, aucun lien ne peut être établi entre la catégorie du 
mot et la fréquence d'apparition des bégayages en situation de lecture en français $(\mathrm{p}=.292)$. Signalons également que les mots lexicaux sont plus longs, dans la mesure où ils étaient composés de 1,701 syllabes en moyenne $(\mathrm{ET}=0,129, \min =$ $1, \max =4)$ tandis que tous les mots grammaticaux n'étaient constitués que d'une seule syllabe $(\mathrm{ET}=0)$.

La deuxième partie de l'étude morphosyntaxique a porté sur l'identification des parties du discours pouvant être en lien avec la fréquence des bégayages. Comme le montre la Figure 1, les pronoms ne sont bégayés qu'à hauteur de $4 \%$. Il en va de même pour les substantifs, dont le taux de bégayage atteint $3,3 \%$. Les déterminants, quant à eux, sont touchés dans $4,4 \%$ des cas. En revanche, les adverbes sont bégayés à hauteur de $16,3 \%$, représentant ainsi la catégorie la plus problématique en lecture pour les locuteurs francophones. Les prépositions sont la deuxième classe grammaticale la plus disfluente avec 12,5\% d'occurrences bégayées. $10 \%$ des conjonctions ont également été porteuses d'un bégayage. Enfin, les verbes et les adjectifs sont touchés à hauteur de $9,5 \%$ et $8,3 \%$ respectivement $(\mathrm{p}=.003)$.

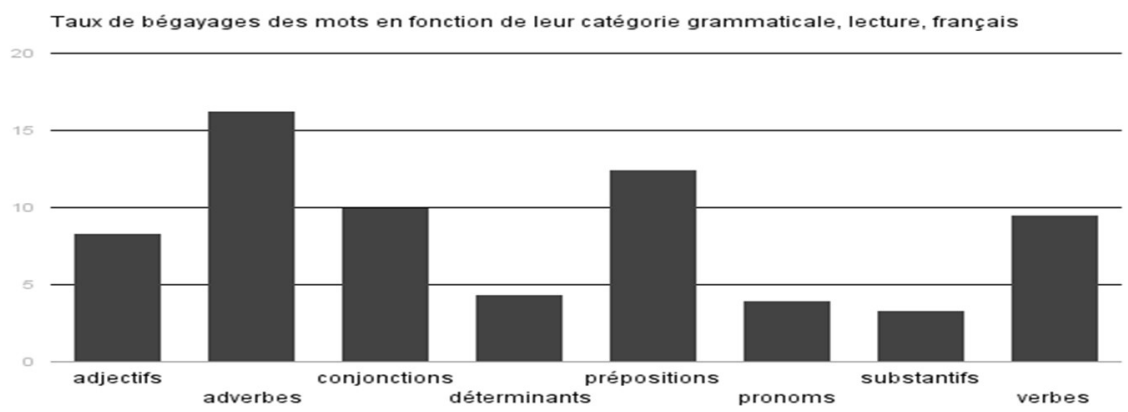

Figure 1 : Taux de bégayages des mots en fonction de leur catégorie grammaticale, lecture, français, en \%

\subsubsection{Parole spontanée}

Sur un total de 7390 mots analysés, 3921 ont été catégorisés comme grammaticaux et 3469 comme lexicaux. 379 mots grammaticaux ont fait objet d'une disfluence sévère, soit 9,7\%, contre 294 mots lexicaux, représentant $8,5 \%$ des cas $(\mathrm{p}=.000)$. Ainsi, les mots-outils semblent être plus problématiques pour cette catégorie de locuteurs en situation de parole spontanée, comme le montre la Figure 2. 


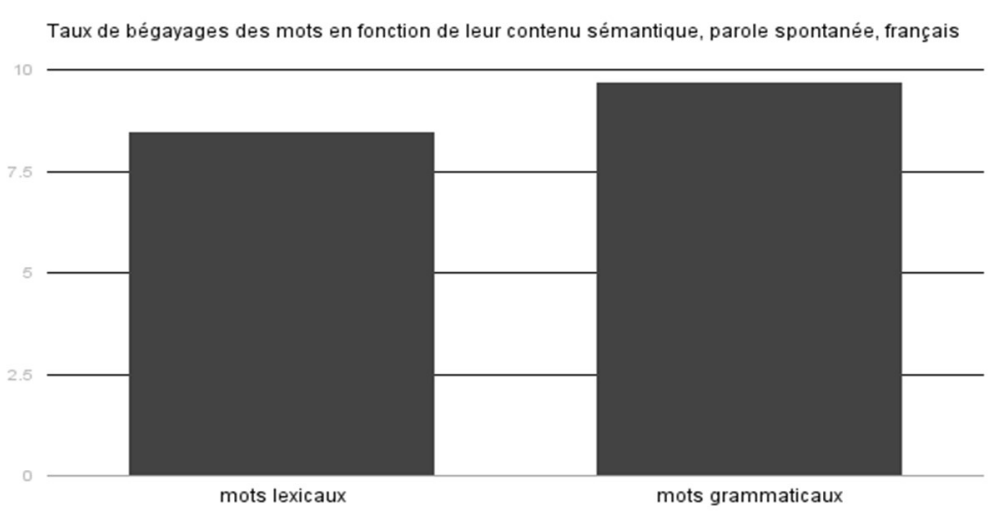

Figure 2 : Taux de bégayages des mots en fonction de leur contenu sémantique, parole spontanée, français, en \%

Par la suite, nous avons défini le nombre d'occurrences de classes grammaticales bégayées par rapport aux occurrences prononcées durant la tâche de parole spontanée en français. Nous observons sur la Figure 3 que la partie du discours la plus problématique pour les PQB francophones est celle des déterminants, dont $12 \%$ sont disfluents. Suivent les pronoms $(10,8 \%)$, les substantifs $(10,6 \%)$ et les prépositions $(10,8 \%)$. 8,8\% de toutes les conjonctions ont été disfluentes. Les verbes et les adjectifs sont respectivement bégayés dans 7,8\% et $7,7 \%$ des cas. Enfin, les interjections sont porteuses d'un bégayage à hauteur de $6 \%$ et les adverbes représentent la catégorie la moins problématique avec 5,4\% d'occurrences bégayées $(\mathrm{p}=.000)$.

Taux de bégayages des mots en fonction de leur catégorie grammaticale, parole spontanée, français

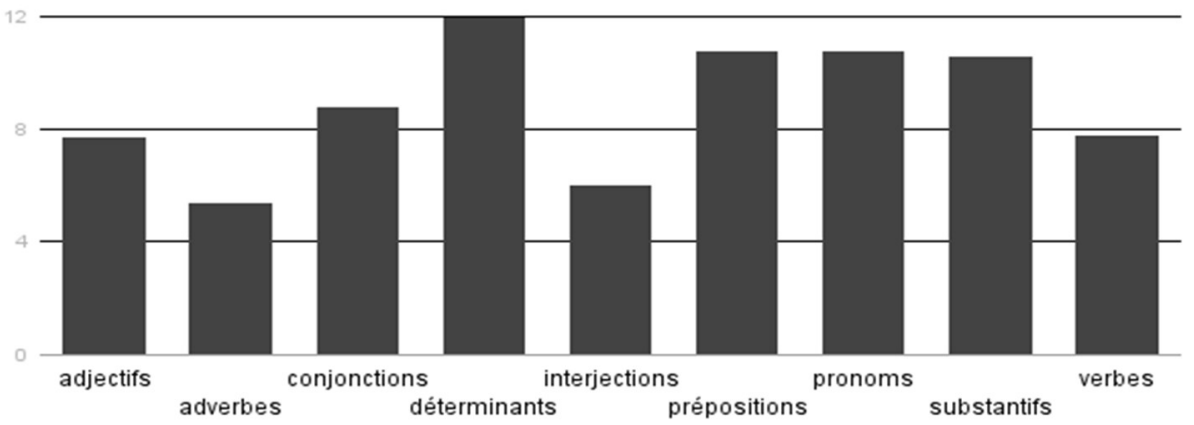

Figure 3 : Taux de bégayages des mots en fonction de leur catégorie grammaticale, parole spontanée, français, en \% 


\subsection{Slovaque}

\subsubsection{Lecture}

Le texte soumis aux locuteurs slovacophones comprend 37 mots grammaticaux et 48 mots lexicaux, soit 85 occurrences au total. Ceci signifie que 850 mots ont été lus par les 10 locuteurs.

Nous avons recensé 172 bégayages en lecture. Ces bégayages ont été distribués comme suit : 34 ont été présents sur un mot grammatical et 138 sur un mot lexical.

De fait, les mots grammaticaux ont été bégayés à hauteur de $9,2 \%(\mathrm{ET}=8,753$; $\min =0, \max =18,919)$, tandis que le taux de disfluences sévères sur les mots lexicaux atteint 28,8\% $(\mathrm{ET}=29,031 ; \min =0, \max =68,75)$. Les résultats sont statistiquement significatifs $(\mathrm{p}=.000)$ : les mots lexicaux semblent donc subir davantage de disfluences que les mots grammaticaux chez les locuteurs slovacophones en tâche de lecture (Figure 4).

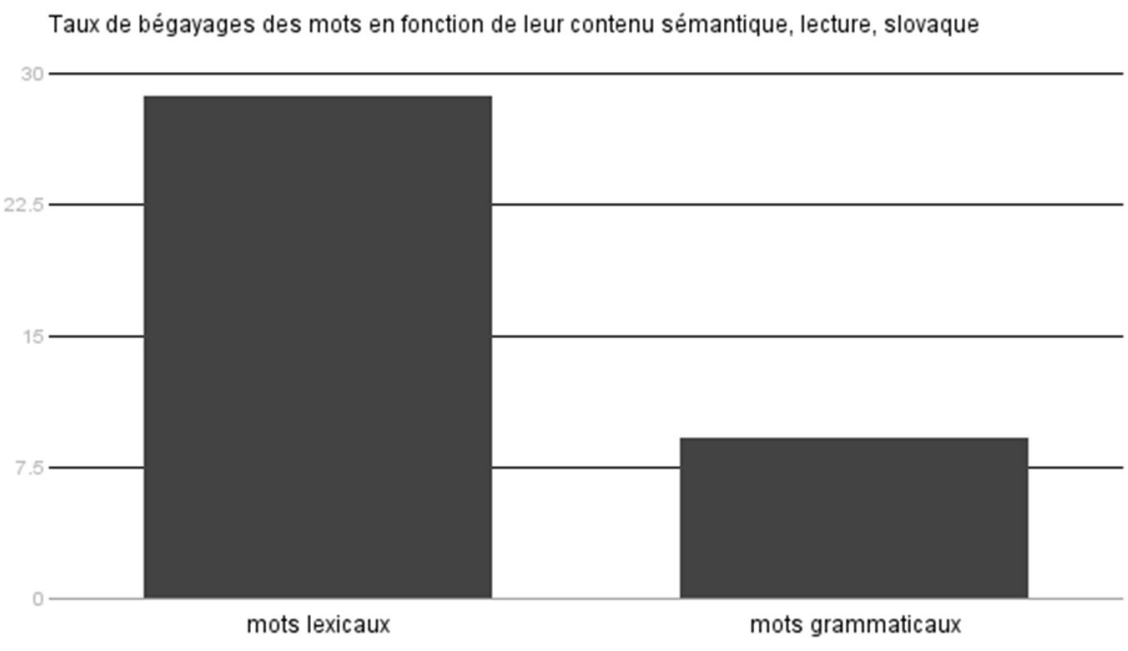

Figure 4 : Taux de bégayages des mots en fonction de leur contenu sémantique, lecture, slovaque, en $\%$

Pour ce qui est des classes grammaticales, comme l'illustre la Figure 5, les verbes sont la catégorie la plus bégayée, puisque $32,4 \%$ des verbes prononcés ont été bégayés. En revanche, si les pronoms constituent la deuxième catégorie grammaticale la plus représentée dans le texte, seuls $6,5 \%$ de ces derniers sont disfluents. $30 \%$ de tous les adjectifs prononcés ont été bégayés, suivis des substantifs, disfluents à hauteur de $24,705 \%$. Les particules et les adverbes semblent également problématiques pour les locuteurs slovacophones, avec respectivement $23,3 \%$ et $20 \%$ d'occurrences bégayées. $11,7 \%$ de prépositions prononcées ont été disfluentes. Enfin, les conjonctions ainsi que les pronoms présentent un taux de bégayages inférieur à $10 \%(8,8 \%$ et $6,5 \%$ respectivement $)$. Ces résultats sont statistiquement significatifs $(\mathrm{p}=.003)$. 


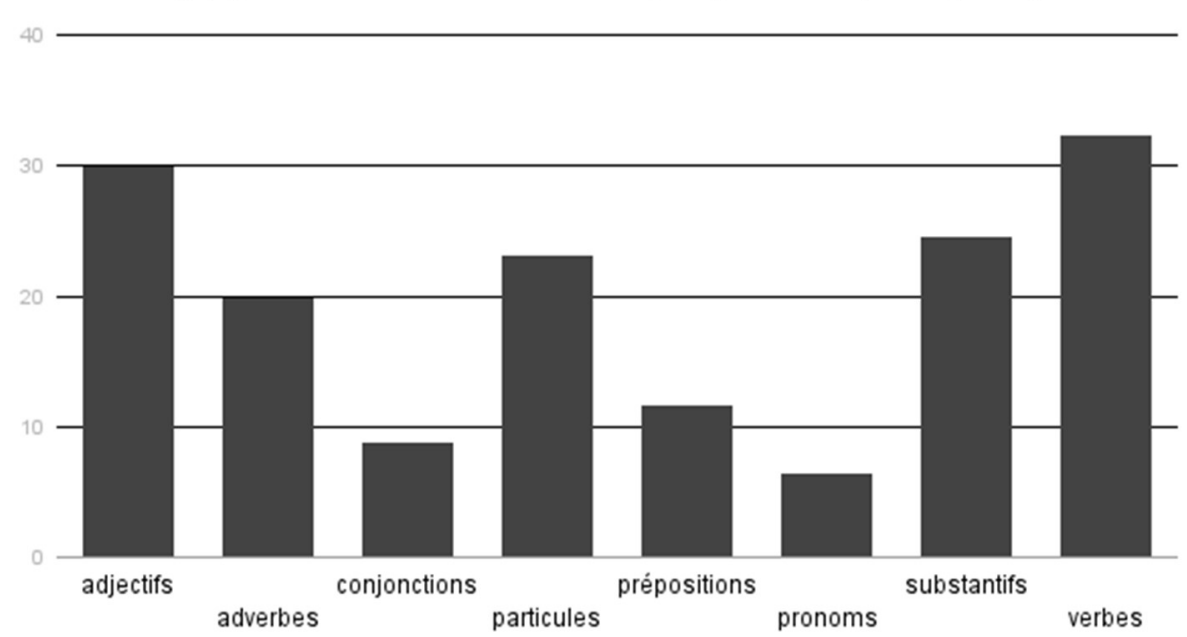

Figure 5 : Taux de bégayages des mots en fonction de leur catégorie grammaticale, lecture, slovaque, en $\%$

\subsubsection{Parole spontanée}

Durant les entretiens, 4994 mots ont été énoncés par les locuteurs slovacophones, dont 2771 mots grammaticaux et 2223 mots lexicaux.

Les mots grammaticaux sont bégayés à hauteur de 7,615\% $(\mathrm{ET}=7,314 ; \mathrm{min}=$ 1,$685 ; \max =24,49$ ), dans la mesure où l'on a relevé 211 disfluences sur les 2771 mots grammaticaux présents dans les discours des sujets enregistrés. Sur 2223 mots lexicaux prononcés, 381 sont bégayés $(17,139 \% ; \mathrm{ET}=15,336 ; \min =1,18 ; \max =$ $52,402)$. Parallèlement aux résultats obtenus dans la tâche de lecture chez ce même groupe de locuteurs, les mots lexicaux semblent davantage enclins à être disfluents que les mots grammaticaux $(p=.000)$ en slovaque (Figure 6). 
Taux de bégayages des mots en fonction de leur contenu sémantique, parole spontanée, slovaque

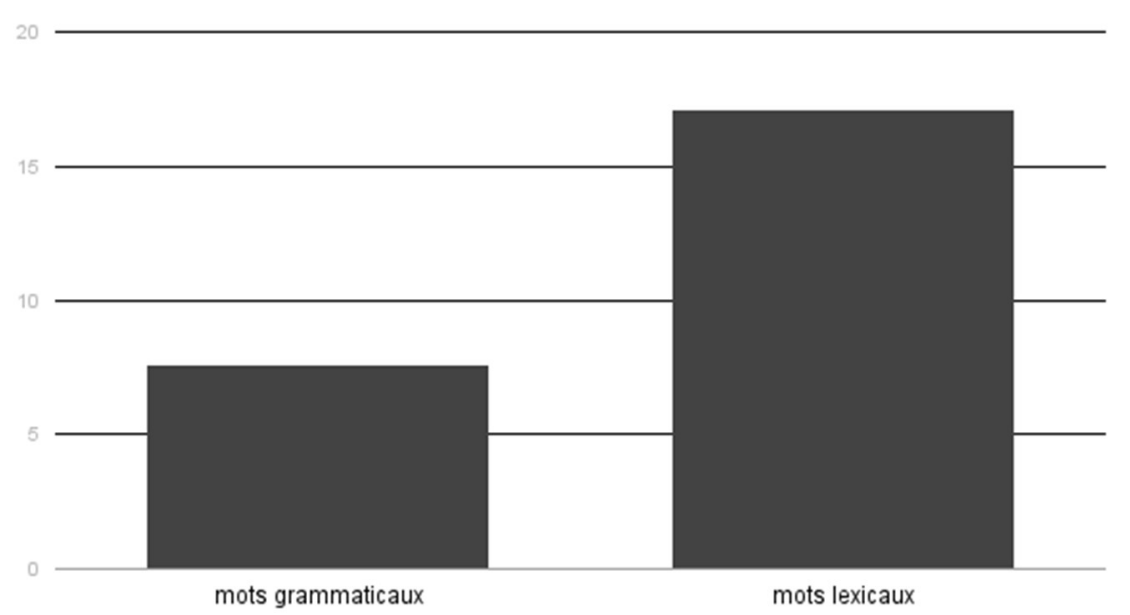

Figure 6 : Taux de bégayages des mots en fonction de leur contenu sémantique, parole spontanée, slovaque, en \%

En ce qui concerne les classes grammaticales en parole spontanée, ce sont les adjectifs qui semblent les plus bégayés dans la mesure où $21,5 \%$ de ces derniers sont disfluents (Figure 7). Suivent les substantifs (17,1\%), les verbes (17\%), les adverbes $(14,6 \%)$, les particules $(11,9 \%)$ et les prépositions $(11 \%)$, tous bégayés à plus de $10 \%$. Inversement, aucune interjection n'a subi de disfluence. Les conjonctions étaient bégayées à hauteur de 5,2\%. Enfin, $6,4 \%$ de pronoms ont subi un bégayage $(\mathrm{p}=.000)$. 
Taux de bégayages des mots en fonction de leur catégorie grammaticale, parole spontanée, slovaque

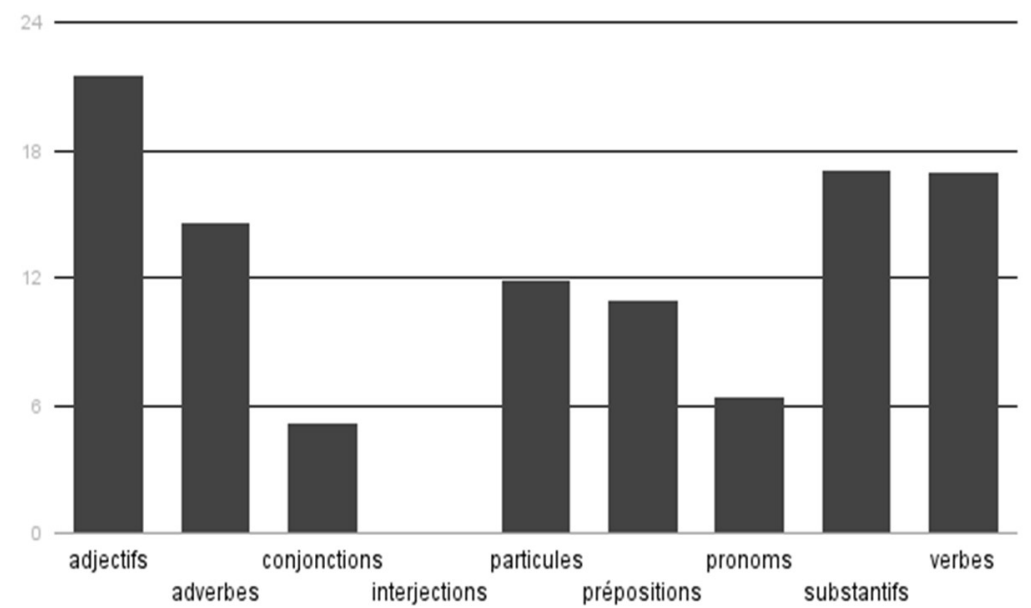

Figure 7 : Taux de bégayages de mots en fonction de leur catégorie grammaticale, parole spontanée, slovaque, en \%

\section{Comparaison entre la lecture et la parole spontanée}

Comme il a été possible de le constater, les résultats ne sont pas les mêmes en tâches de lecture et de parole spontanée. C'est la raison pour laquelle nous avons comparé ces deux exercices.

\subsection{Français}

La comparaison des éléments les plus bégayés en lecture et en parole spontanée montre que ce sont les adverbes qui sont les plus disfluents dans le premier cas et les déterminants dans le second. La catégorie la plus bégayée devance de près de 7 points la deuxième en lecture, tandis qu'en parole spontanée, ce déséquilibre s'efface avec 1,35 point seulement entre la première et la quatrième catégorie la plus touchée (Figure 8). 


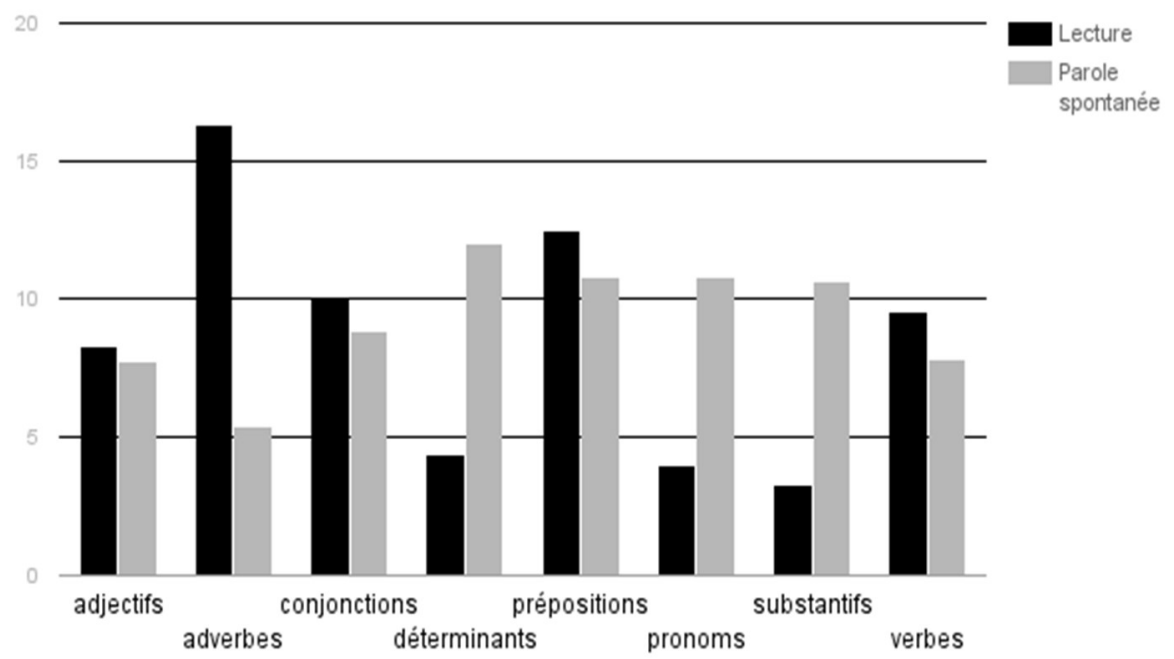

Figure 8 : Taux de bégayages des mots en fonction de leur catégorie grammaticale, parole lue et parole spontanée, français

\subsection{Slovaque}

Les bégayages produits par les sujets slovacophones semblent survenir approximativement sur les mêmes parties du discours. Ainsi, aussi bien en parole lue qu'en parole spontanée, les adjectifs, les substantifs et les verbes font partie des trois catégories grammaticales les plus bégayées. Inversement, les conjonctions et les pronoms sont les catégories les moins disfluentes, aussi bien en tâche de lecture qu'en parole spontanée (Figure 9).

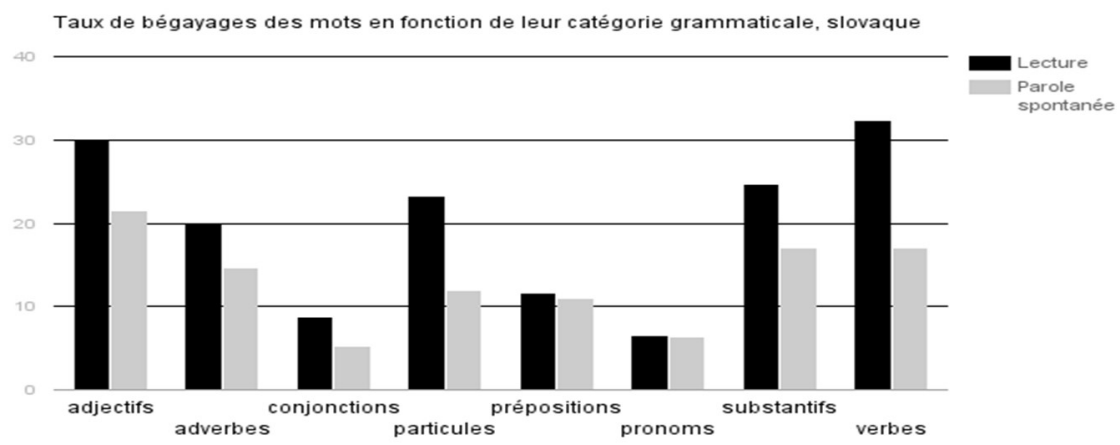

Figure 9 : Taux de bégayages des mots en fonction de leur catégorie grammaticale, parole lue et parole spontanée, slovaque, en \%

\section{Discussion}

L'objectif de cette recherche était de vérifier s'il existe un lien entre les disfluences perçues comme typiques du bégaiement et la classe grammaticale des 
mots dans lesquels elles apparaissent. Nous avons pour cela étudié les productions orales lues et spontanées de locuteurs adultes qui bégaient et ce, dans deux langues différentes, à savoir le français et le slovaque.

Les données obtenues pour le français sur la catégorie des mots bégayés n'ont pas permis de confirmer ou d'infirmer les observations effectuées pour d'autres langues en tâche de lecture. En revanche, le taux de bégayages était plus élevé sur les mots-outils que sur les mots lexicaux en parole spontanée. Ce résultat, qui ne valide pas les observations faites pour d'autres langues, pourrait s'expliquer par les stratégies d'évitement employées par les PQB. La tâche de parole spontanée n'imposant aucune structure au locuteur, ce dernier a la possibilité d'éviter les éléments dont il sait qu'ils lui sont problématiques.

En ce qui concerne les catégories grammaticales les plus bégayées, nous avons observé des résultats différents en tâche de lecture et en parole spontanée chez les locuteurs francophones. En lecture, les adverbes, les prépositions et les verbes ont été relevés comme les plus problématiques. Les déterminants, les prépositions et les substantifs sont, quant à eux, les plus touchés en parole spontanée. De fait, il semblerait que pour ces sujets, le fait d'avoir ou non un texte imposé générerait des difficultés de préparation et d'exécution motrices qui ne seraient pas équivalentes. Parmi les mots-outils, ce sont les prépositions qui sont les plus altérées en lecture, alors que les taux de bégayages semblent davantage équilibrés en parole spontanée ; on relèvera toutefois une fréquence de bégayages légèrement plus importante pour les déterminants. Concernant les mots lexicaux, ce sont les adverbes en parole lue et les substantifs en parole spontanée qui sont les plus disfluents. Ces divergences laissent penser que le type d'effort cognitif aurait une influence sur le bégaiement : lorsque le sujet n'a pas à définir ses unités lexicales, sa structure et son rythme, les difficultés de prononciation s'en trouveraient modifiées. Rappelons toutefois que du fait de taux de bégayages globalement faibles en tâche de lecture pour ces locuteurs, il serait souhaitable de reprendre cette recherche avec d'autres participants atteints d'un bégaiement plus sévère, afin d'obtenir des résultats statistiquement plus robustes.

Chez les locuteurs slovacophones, nous avons pu constater des taux de bégayages comparables dans les deux tâches demandées. En outre, le nombre d'occurrences disfluentes a été suffisamment élevé pour donner lieu à des résultats significatifs aussi bien en lecture qu'en parole spontanée.

Nous avons également relevé un taux de bégayages plus élevé sur les mots lexicaux que sur les mots-outils, venant ainsi confirmer les résultats obtenus pour l'anglais, l'allemand ou le turc (BUHR \& ZEBROWSKI, 2009 ; DOWRZYNSKI et al., 2003 ; OZDEMIR et al., 2015). La structure linguistique étant différente en slovaque par rapport aux autres langues citées, il serait dès lors souhaitable d'approfondir cette étude en nous intéressant à d'autres paramètres observables pour les mots bégayés : l'accentuation, le nombre de syllabes, la structure phonologique, mais aussi les contextes pragmatique ou sémantique pourraient être analysés afin de vérifier si d'autres critères linguistiques peuvent expliquer l'apparition d'une disfluence.

En nous intéressant ensuite aux catégories grammaticales, nous relevons que les substantifs, les adjectifs et les adverbes constituent les classes les plus disfluentes 
chez les locuteurs slovacophones dans les deux situations. A l'inverse, les conjonctions et les pronoms étaient les catégories les moins problématiques, aussi bien en tâche de lecture qu'en parole spontanée. Ainsi, il semblerait que lorsque les taux de bégayages sont plus équilibrés dans les deux tâches, les difficultés resteraient plus ou moins comparables.

\section{Conclusion}

La présente étude a permis de relever un certain nombre de différences interlangues dans l'emplacement des disfluences chez les locuteurs qui bégaient. Ces différences peuvent être expliquées par un certain nombre de dissimilitudes, notamment au niveau de la fréquence de bégayages en situation de lecture. Une autre explication possible viendrait des structures syntaxiques (plus ou moins libres selon la langue) et phonologiques (accentuation, phonotactique, etc.), qui ne sont pas les mêmes en français et en slovaque. Si l'ensemble de ces éléments restent à vérifier, un tel constat permettrait de mieux appréhender les éléments les plus problématiques dans chaque langue afin d'adapter la prise en charge orthophonique.

\section{Remerciements}

Cette recherche a été en partie financée par le Défi Instrumentation aux limites SYNABE.

\section{BIBLIOGRAPHIE}

ABDALLA Fauzia ; RoBB Michael, AL-SHATTI Tareq (2010), Stuttering and Lexical Category in Adult Arabic Speakers, Clinical Linguistics \& Phonetics 24(1), p. 70-8.

Au-Yeung James ; VAllejo Gomez Isabel, Howell Peter (2003), Exchange of Disfluency With Age From Function Words to Content Words in Spanish Speakers Who Stutter, Journal of Speech, Language, and Hearing Research 46, p. 754-765.

BLANCHE-BENVENISTE Claire (1991), Les études sur l'oral et le travail d'écriture de certains poètes contemporains, Langue française 89 (1), p. 52-71.

BLOODSTEIN Oliver; GANTWERK, Barbara (1967), Grammatical Function in Relation to Stuttering in Young Children, Journal of Speech, Language, and Hearing Research 10, p. 786-789.

BUHR Anthony; ZeBROWSKI Patricia (2009), Sentence Position and Syntactic Complexity of Stuttering in Early Childhood: a Longitudinal Study, Journal of Fluency Disorders 34(3), p. 155-172.

DIDIRKOVA Ivana (2016), Parole, langues et disfluences : Une étude linguistique et phonétique du bégaiement, Thèse de doctorat, Montpellier, Université PaulValéry Montpellier 3.

DIDIRKOVÁ Ivana; FAUTH Camille; HIRSCH Fabrice; LUXARDO Giancarlo; DIWERSY Sascha (2016), Disfluences normales vs. Disfluences sévères : une étude acoustique, Actes de la conférence conjointe JEP-TALN-RECITAL 2016 vol. 1 : JEP, p. 563-570. 
DwORZYNSKI Katharina; Howell Peter; NATKe Ulrich (2003), Predicting Stuttering from Linguistic Factors for German Speakers in Two Age Groups, Journal of Fluency Disorders 28(2), p. 95-112.

Howell Peter; AU-YeUng James ; SACKIN Stevie (1999), Exchange of Stuttering From Function Words to Content Words With Age, Journal of Speech, Language, and Hearing Research 42(2), p. 345-354.

MONFRAIS-PFAUWADEL Marie-Claude (2014), Bégaiement, bégaiements. Un manuel clinique et thérapeutique, Paris, De Boeck-Solal.

NATKE Ulrich, SANDRIESER Patricia, VAN ARK Melanie, PIETROWSKY Reinhard, Kalveram Karl (2004), Linguistic Stress, Within-Word Position, and Grammatical Class in Relation to Early Childhood Stuttering, Journal of Fluency Disorders 29(1), p. 109-122.

NEWMAN Rochelle; BERSTEIN RATNER Nan (2007), The Role of Selected Lexical Factors on Confrontation Naming Accuracy, Speed and Fluency in Adults Who Do and Do Not Stutter, Journal of Speech, Language, and Hearing Research 50(1), p. 196-213.

OzDEMIR Sertan; Aydin Ayse ; Selvi Selami (2015), The Relationship between Lexical Category and Stuttering Moments in Turkish, Procedia - Social and Behavioral Sciences 193, p. 351.

PRINS David; MAIN Victoria; WAMPLER Susan (1997), Lexicalization in Adults who Stutter, Journal of Speech, Language and Hearing Research 40, p. 373384.

QUARRINGTON Bruce ; CONWAY Jerome ; SIEGEL Nathan (1962), An Experimental Study of Some Properties of Stuttered Words, Journal of Speech, Language, and Hearing Research 5, p. 387-394.

ROMMEL Dieter (2001), The Influence of Psycholinguistic Variables on Stuttering in Childhood, in: BosshardT Hans-Georg; YARUSS J. Scott; Peters Hermann F.M. (éd.), Fluency Disorders: Theory, Research, Treatment and Selfhelp, Proceedings of The Third World Congress of Fluency Disorders in Nyborg, Denmark, Nijmegen, Nijmegen University Press, p. 195-202.

Rose Yvan, MACWHINNEY Brian, BYRNE Rodrigue, HEDLUND Gregory, MADDOCKS Keith, O'BRIEN Philip, WAREHAM Todd (2006), Introducing Phon: a Software Solution for the Study of Phonological Acquisition, Proceedings of the 30th Boston University Conference on Language Development, p. 489-500.

SCHMID Helmut (1995), Improvements in Part-of-Speech Tagging with an Application to German, Proceedings of the ACL SIGDAT-Workshop, Dublin, Ireland.

StARKWEATHER C. Woodruff (1987), Fluency and Stuttering, Englewood Cliffs, N.J.: Pretince-Hall Inc.

WiNGATE Marcel E. (1967), Stuttering and Word Length, Journal of Speech, Language, and Hearing Research 10, p. 146-152. 